\title{
Post-stimulus endogenous and exogenous oscillations are differentially modulated by task difficulty
}

\author{
Yun $\mathrm{Li}^{1,2}$, Bin Lou ${ }^{2}$, Xiaorong Gao ${ }^{1}$ and Paul Sajda ${ }^{2 *}$ \\ Department of Biomedical Engineering, Tsinghua University, Beijing, China \\ 2 Department of Biomedical Engineering, Columbia University, New York, NY, USA
}

\author{
Edited by: \\ John J. Foxe, Albert Einstein College \\ of Medicine, USA \\ Reviewed by: \\ Simon P. Kelly, City College of New \\ York, USA \\ Manuel Gomez-Ramirez, The Johns \\ Hopkins University, USA \\ Kevin Whittingstall, Université de \\ Sherbrooke, Canada \\ *Correspondence: \\ Paul Sajda, Department of \\ Biomedical Engineering, Columbia \\ University, 351 Engineering Terrace \\ Building, Mail Code 8904, 1210 \\ Amsterdam Avenue, New York, \\ NY 10027, USA \\ e-mail:psajda@columbia.edu
}

We investigate the modulation of post-stimulus endogenous and exogenous oscillations when a visual discrimination is made more difficult. We use exogenous frequency tagging to induce steady-state visually evoked potentials (SSVEP) while subjects perform a face-car discrimination task, the difficulty of which varies on a trial-to-trial basis by varying the noise (phase coherence) in the image. We simultaneously analyze amplitude modulations of the SSVEP and endogenous alpha activity as a function of task difficulty. SSVEP modulation can be viewed as a neural marker of attention toward/away from the primary task, while modulation of post-stimulus alpha is closely related to cortical information processing. We find that as the task becomes more difficult, the amplitude of SSVEP decreases significantly, approximately $250-450 \mathrm{~ms}$ post-stimulus. Significant changes in endogenous alpha amplitude follow SSVEP modulation, occurring at approximately 400-700 ms post-stimulus and, unlike the SSVEP, the alpha amplitude is increasingly suppressed as the task becomes less difficult. Our results demonstrate simultaneous measurement of endogenous and exogenous oscillations that are modulated by task difficulty, and that the specific timing of these modulations likely reflects underlying information processing flow during perceptual decision-making.

Keywords: alpha oscillations, face perception, electroencephalography (EEG), SSVEP, attention, perceptual decision-making

\section{INTRODUCTION}

Neural oscillations, for example measured via electroencephalography (EEG), have been studied for decades in an effort to link brain state to perceptual and cognitive processing. Endogenous oscillations are attributable to internal neural processes and include a well-known set of frequencies ranging from the low delta to the high gamma band (Niedermeyer and Lopes da Silva, 1993). Exogenous oscillations are driven by the rhythms of external stimuli and are typically associated with sensory systems [e.g., steady state visually evoked potentials (SSVEP) and auditory steady state response (ASSR)] (Picton et al., 2003; Vialatte et al., 2010 review).

A prominent endogenous brain rhythm is the alpha oscillation which has been extensively investigated within the context of both pre-stimulus and post-stimulus effects (Babiloni et al., 2006; Hanslmayr et al., 2007; Freunberger et al., 2008; van Dijk et al., 2008). The neural mechanism underlying alpha oscillations also have been deeply explored (Lopes da Silva, 1991). Though alpha activity is often thought to represent an idling or inattentive state (Pfurtscheller et al., 1996 review), some studies suggest that it also reflects a suppression mechanism of irrelevant information (Worden et al., 2000; Kelly et al., 2006; Rihs et al., 2007; Foxe and Snyder, 2011; Gomez-Ramirez et al., 2011), and/or an inhibition of information processing (Klimesch et al., 1997, 2007 review). Specifically, previous studies of post-stimulus alpha activity within the context of visual object recognition have shown that alpha desynchronization was greater for the recognition of meaningful objects than it was for meaningless objects (Klimesch et al., 1997; Vanni et al., 1997; Mima et al., 2001; Freunberger et al., 2008), suggesting that post-stimulus alpha activity is related to semantic information processing.

SSVEP, an oscillatory brain response evoked by a flickering visual stimulus, is an exogenous form of frequency tagging that has been shown to index the allocation of cognitive resources such as attention (Vialatte et al., 2010 review). Many studies have reported that SSVEP amplitude is decreased when attention must compete or be split between the flicker and a background picture (Müller et al., 2008, 2011; Attar et al., 2010). Moreover, a study by Andersen and Müller in 2010 revealed that the facilitation of SSVEP amplitude for the attended stimulus is accompanied by suppression for the unattended stimulus (Andersen and Müller, 2010). These findings suggested that SSVEPs can be used as neural marker of the time course of attentional resource competition.

In this paper, we aim to simultaneously investigate how poststimulus endogenous and exogenous oscillations are affected as a function of task difficulty during a face/car discrimination task. Specifically, we superimpose a flickering stimulus of $15 \mathrm{~Hz}$ upon a sequence of images and simultaneously analyze the time course and spatial distribution of exogenouslyinduced SSVEPs and endogenous alpha oscillations as a function of image phase coherence. Our results demonstrate that the phase coherence of the stimulus, being our surrogate for the difficulty of the visual discrimination, differentially modulates 
exogenously-induced SSVEPs and endogenous alpha oscillations at different times and that this may reflect underlying information processing flow during the visual discrimination task.

\section{MATERIALS AND METHODS \\ SUBJECTS}

Eleven right-handed subjects (three females and eight males; mean \pm SD age, $26.5 \pm 5.9$ years) with normal or corrected-tonormal vision participated in this study. Informed consent in accordance with the guidelines and approval of the Columbia University Institutional Review Board was obtained from all subjects.

\section{STIMULI AND EXPERIMENTAL PROCEDURE}

Stimuli were presented in the center of a $17^{\prime}$ LCD monitor with a refresh rate of $60 \mathrm{~Hz}$. A set of 17 face (Max Planck Institute face database; Troje and Bülthoff, 1996) and 17 car grayscale images (image size, $512 \times 512$ pixels; 8 bits/pixel) were used. The car image database was the same used in Philiastides and Sajda (2006) and was constructed by taking images from the internet, segmenting the car from the background in each, converting the image to grayscale and then resizing it to be comparable in size to the face images. The pose of the faces and cars was also matched across the entire database and was sampled at random (left, right, center). Equal luminance and contrast were used. All images had identical magnitude spectra (average magnitude spectrum of all images in the database), and their corresponding phase spectra were manipulated using the weighted mean phase (WMP) (Dakin et al., 2002) technique to generate a set of images characterized by their percentage of phase coherence. In this experiment, four different phase coherence levels $(30,35,40$, and $45 \%$ ) were utilized to create tasks of different difficulty levels (Figure 1B).

The schematic of the experimental design is shown in Figure 1A. Face/car (i.e., "task") images were presented for $50 \mathrm{~ms}$ followed by a random inter-stimulus interval (ISI) uniformly distributed between 2000 and 3000 ms. During the ISI, a uniform grayscale image was presented as the background, which had the same size and average grayscale value as the task images. Frequency tagging was done by superimposing a total of 900 randomly-placed small white squares (each $3 \times 3$ pixels) on the stream of images (tagging was continuous across the task images and ISI), with the white squares having a flicker frequency of $15 \mathrm{~Hz} .15 \mathrm{~Hz}$ was chosen so that endogenous alpha power could also be measured. The ISIs was randomly generated after each trial, and task images were not phase-locked to the $15 \mathrm{~Hz}$ flicker, which effectively reduced the interaction between the event related potentials (ERPs) and SSVEPs.

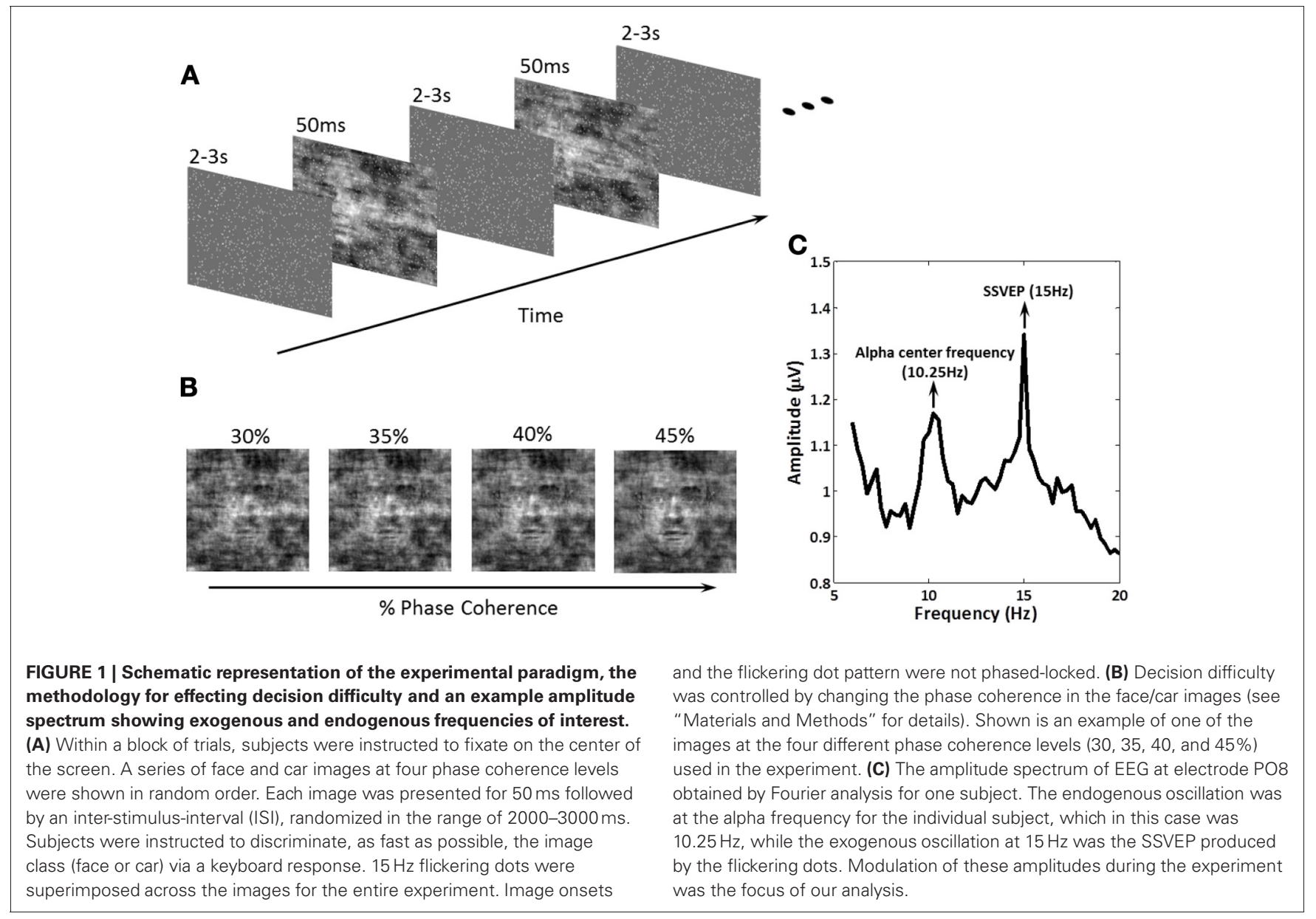


Subjects were instructed to discriminate task images as rapidly as possible. Each experimental block consisted of 34 trials of face and car images at each of four different phase coherence levels, with images presented randomly within a block. In each experiment, there were six blocks in total. When a task image appeared, subjects reported their decision regarding the category of the image by pressing a button on the keyboard, with the left arrow key for cars and right arrow key for faces, using one of two fingers on their right hands. Subjects were instructed to maintain central fixation throughout the entire experiment and to respond as quickly and accurately as possible.

\section{DATA ACQUISITION}

Participants were seated in an electrostatically shielded room and positioned at a $1 \mathrm{~m}$ distance from the screen. EEG was recorded using a Sensorium 84-channel Ag/AgCl electrode system (Sensorium Inc., Vermont, USA). The ground channel was located between the eyebrows, and all channels were referenced to the left mastoid. All impedances were below $20 \mathrm{k} \Omega$ and the sampling rate was $1000 \mathrm{~Hz}$. Stimulus events and motor responses were recorded on separate channels.

\section{DATA PROCESSING}

Epochs were extracted according to the task events. Trials with strong eye movements or other movement artifacts were manually rejected, resulting in less than 20\% trials rejected. Only EEG from correct trials with reaction times below $1000 \mathrm{~ms}$ was analyzed. During the behavioral and EEG data analysis, face and car trials at the same coherence level were considered equal in difficulty. In other words, there were only four difficulty conditions, corresponding to the four phase coherence levels.

For every subject, decision accuracy and mean reaction time from correct trials at each phase coherence level were calculated. To test the consistency of behavioral performance across subjects, a balanced One-Way ANOVA, testing the effect of phase coherence levels $(30,35,40$, and 45\%), was performed. Also, paired $t$-tests were performed between each pair of phase coherence levels.

Since SSVEP is primarily seen in visual cortex (Regan, 1989; Vialatte et al., 2010 review) and alpha oscillations were predominantly found at parietal-occipital areas (Adrian and Matthews, 1934; Palva and Palva, 2007), we re-referenced the EEG to electrode $\mathrm{Fz}$ since it is distant from visual cortex. The amplitude spectrum of EEG waveforms at electrode PO 8 obtained by Fourier analysis (Figure 1C) illustrates the SSVEP at $15 \mathrm{~Hz}$ relative to the alpha power. We confirmed that each subject's central alpha frequency is well separated from the exogenous oscillation at $15 \mathrm{~Hz}$ induced by the flickering stimuli.

The time course of SSVEP amplitude at each phase coherence level was quantified by the following steps.

1. Narrow band pass filtering was done using a zero-phase filter within the range of $15 \pm 1.6 \mathrm{~Hz}$ to isolate the SSVEP signal.

2. Calculating the analytic signal of the filtered EEG by applying the Hilbert transform.

3. Estimating the instantaneous SSVEP amplitude from the complex amplitude of the analytic signal.

4. Normalizing the instantaneous SSVEP amplitude by subtracting the averaged amplitude of a baseline from -200 to $0 \mathrm{~ms}$ before the target and then dividing by the same baseline (Andersen and Müller, 2010).

Specially, we assumed the baselines of all conditions are the same, and therefore only calculated one baseline for each subject by averaging all trials across all conditions. The result is the normalized instantaneous SSVEP amplitude reflecting the changes in SSVEP amplitude relative to the baseline, which ensures that each subject contributes, more or less, equally to the average, avoiding the group results from being dominated by a single subject.

Evidence from EEG, magnetoencephalography (MEG) and fMRI suggests the existence of face discriminating activity in right lateral occipital cortex (rLOC, near electrode PO8) (Bentin et al., 1996; Jeffreys, 1996; Kanwisher et al., 1997; Liu et al., 2000), while a study by Philiastides and Sajda (2007) demonstrated a connection between the LOC and decision difficulty. To identify electrodes that were most relevant to both discrimination and difficulty in the task, we analyzed the spatial distributions of the ERP amplitude differences at $170 \mathrm{~ms}$ post-stimulus between face and car trials at the $45 \%$ phase coherence level (Figure 2A), as well as at $220 \mathrm{~ms}$ post-stimulus comparing the 30 and $45 \%$ phase coherence levels (Figure 2B). This approach was taken since previous studies have demonstrated that the difference between categories was characterized by the amplitude difference of the N170 component, while the difficulty effect was quantified by the amplitude difference of the D220 component (Philiastides and Sajda, 2006; Philiastides et al., 2006). Our primary analysis of endogenous and exogenous frequency modulations was then done on the electrode with the most significant selectivity for face vs. car and task difficulty (i.e., sensitivity to phase coherence level). This turned out to be electrode PO8. Additional analysis showing the spatial distribution of the modulations across all electrodes is reported in Figures $\mathbf{5}$ and $\mathbf{7}$.

To identify time periods in which phase coherence had a significant effect, we performed a set of statistical tests on the normalized SSVEP amplitude. First, paired $t$-tests between phase coherence levels of 30 and $45 \%$ were conducted over each time point from 0 to $800 \mathrm{~ms}$ post-stimulus on all electrodes. Next, we adopted a modified cluster-analysis approach to correct for

A

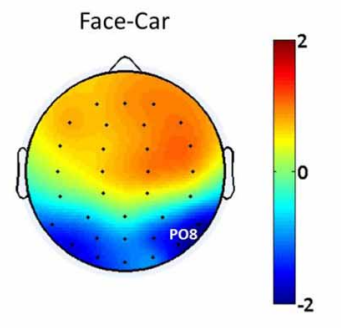

FIGURE 2 | Spatial distributions of ERP amplitude differences between (A) face and car trials at $\mathbf{1 7 0} \mathrm{ms}$ post-stimulus, (B) phase coherence levels of 45 and $30 \%$ at $220 \mathrm{~ms}$ post-stimulus. 
multiple comparisons and identify time periods within which the SSVEP amplitude between phase coherence levels was significantly different. Specifically, the time points over which the null hypothesis was rejected at a significance level of 0.05 were selected and clustered based on their temporal adjacency. The maximum temporal period across all contiguous clusters were used as for constructing the cluster-level statistics. The data were then randomized across two phase coherence levels (30 and 45\%) to generate shuffled cluster-level statistics. We performed all possible permutations of the 11 subjects to generate the shuffled cluster-level statistics. Finally, corrected $p$-values were calculated by comparing the values of the cluster-level statistics of the original data against the distribution of the shuffled cluster-level statistics across permutations (Maris and Oostenveld, 2007; Rohenkohl and Nobre, 2011). Subsequently, the time periods with corrected p-values less than 0.05 at electrode PO8 were selected for analyzing the spatial distribution of SSVEP amplitude in parietal and occipital areas at each phase coherence level.

The effect of phase coherence on the alpha band was quantified using the same processing steps as the SSVEP analysis, described above, except that the filtering band was specific for each subject, in terms of their alpha center frequency $\pm 1.6 \mathrm{~Hz}$. The mean alpha center frequency across all subjects was $10.5 \mathrm{~Hz}(\mathrm{SD}, 1.0 \mathrm{~Hz})$. Since motor related oscillations ( $\mathrm{mu}$ rhythm) share a common frequency band $(8-12 \mathrm{~Hz})$ with endogenous alpha oscillations, response-locked data of both SSVEP and alpha oscillations were also analyzed to investigate the effect of the motor response.

\section{RESULTS \\ BEHAVIORAL RESULTS}

Averaged behavioral performance across all subjects at each phase coherence level is shown in Figure 3. It is clear that reaction time is increased (Figure $\mathbf{3 A}$ ) and decision accuracy is decreased (Figure 3B) as phase coherence level decreases. A balanced OneWay ANOVA demonstrates a significant effect of phase coherence level on reaction time $\left[F_{(3,36)}=2.94, p<0.05\right]$ and decision accuracy $\left[F_{(3,40)}=17.93, p<0.001\right]$. A set of paired $t$-tests demonstrates that there is a significant difference between any two phase coherence levels for reaction time $(p<0.01)$ and decision accuracy $(p<0.01)$. Behavioral results thus clearly demonstrate a significant effect of phase coherence on decision difficulty as measured via reaction time and accuracy.

\section{EFFECT OF PHASE COHERENCE ON EXOGENOUS OSCILLATIONS}

We tracked the time course of the SSVEP amplitude as a way to explore how exogenous oscillations are modulated by phase coherence and therefore decision difficulty. Figure $4 \mathrm{~A}$ shows the time course of the normalized SSVEP amplitude at electrode PO8. There is a suppression of normalized SSVEP amplitude immediately after stimulus onset. Using a paired $t$-test between phase coherence levels of 30 and $45 \%$, a significant effect of phase coherence $(p<0.05)$ was observed from roughly 266 to $466 \mathrm{~ms}$ post-stimulus. This time period was confirmed to be significant with multiple comparisons correction using cluster-level statistics $(p<0.05)$. The average SSVEP amplitude in this time period at electrode PO8 for each of the four phase coherences is presented in Figure 4B. As phase coherence increases, the average SSVEP amplitude also increases, indicating a greater suppression of SSVEP amplitude at lower phase coherence levels. The average SSVEP amplitudes in this period at phase coherence levels of 30 and $35 \%$ are significantly different from those at a phase coherence level of $45 \%$ (paired $t$-test between 30 and $45 \%$ phase coherence levels: $t_{(10)}=3.16, p=0.010$; paired $t$-test between 35 and $45 \%$ phase coherence levels: $\left.t_{(10)}=2.63, p=0.025\right)$.

The scalp topologies of the average SSVEP amplitude from 266 to $466 \mathrm{~ms}$, for each of the four phase coherence levels, are plotted in Figure 5A. The reduction of SSVEP power is mainly in occipital areas, and this reduction is greater for lower phase coherences. The spatial distribution of the difference in SSVEP amplitude between 30 and 45\% coherence levels (Figure 5B) illustrates that phase coherence/task difficulty effects are substantial in the region near electrode PO8. The $p$-value at each
A

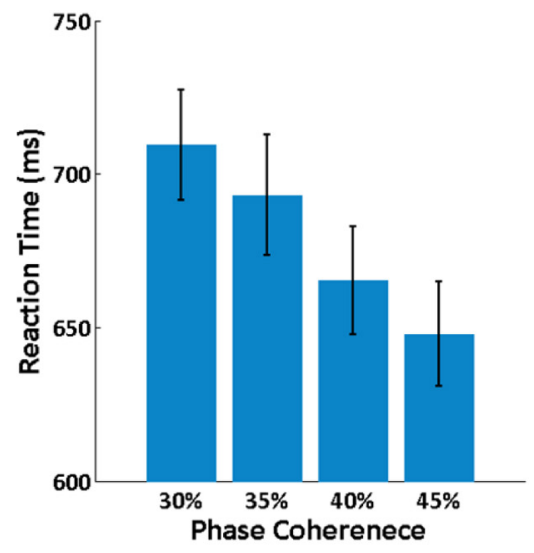

B

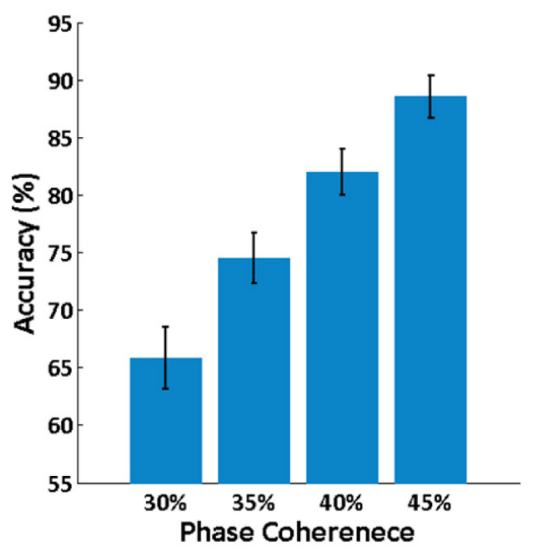

FIGURE 3 | Behavioral results. (A) Mean reaction time averaged over subjects at each phase coherence level. (B) Average decision accuracy across subjects at each phase coherence level. Error bars indicate the standard error. 


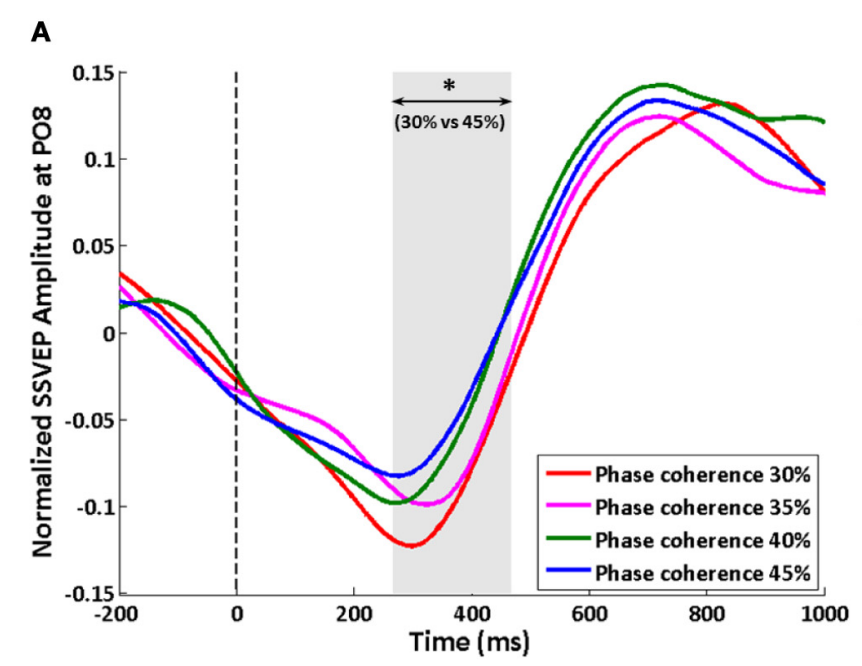

B

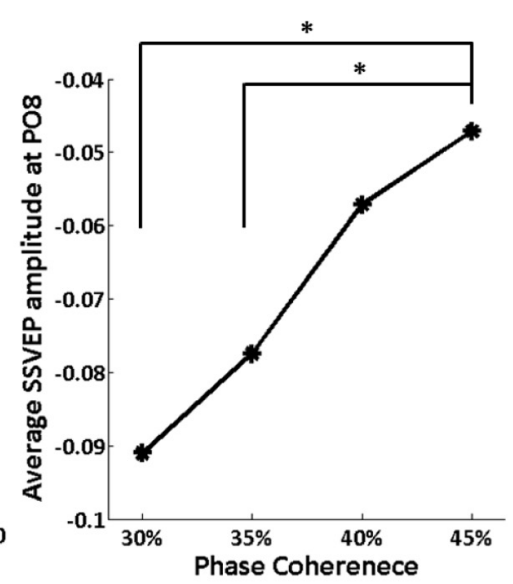

FIGURE 4 | Effect of decision difficulty on exogenous oscillations as measured by normalized SSVEP amplitudes at electrode PO8. (A) Time course of normalized SSVEP amplitude, shown for each of four phase coherence levels. The shaded area indicates the time period (266-466 ms) having a significant difference in normalized SSVEP amplitude between phase coherence levels of 30 and $45 \%$ as assessed by paired $t$-test across subjects and cluster-level statistics $(p<0.05)$. The vertical dashed line indicates the onset of task images. (B) Average SSVEP amplitude from 266 to $466 \mathrm{~ms}$ at each phase coherence level. Asterisks indicate significant differences (paired $t$-test, $p<0.05$ ).
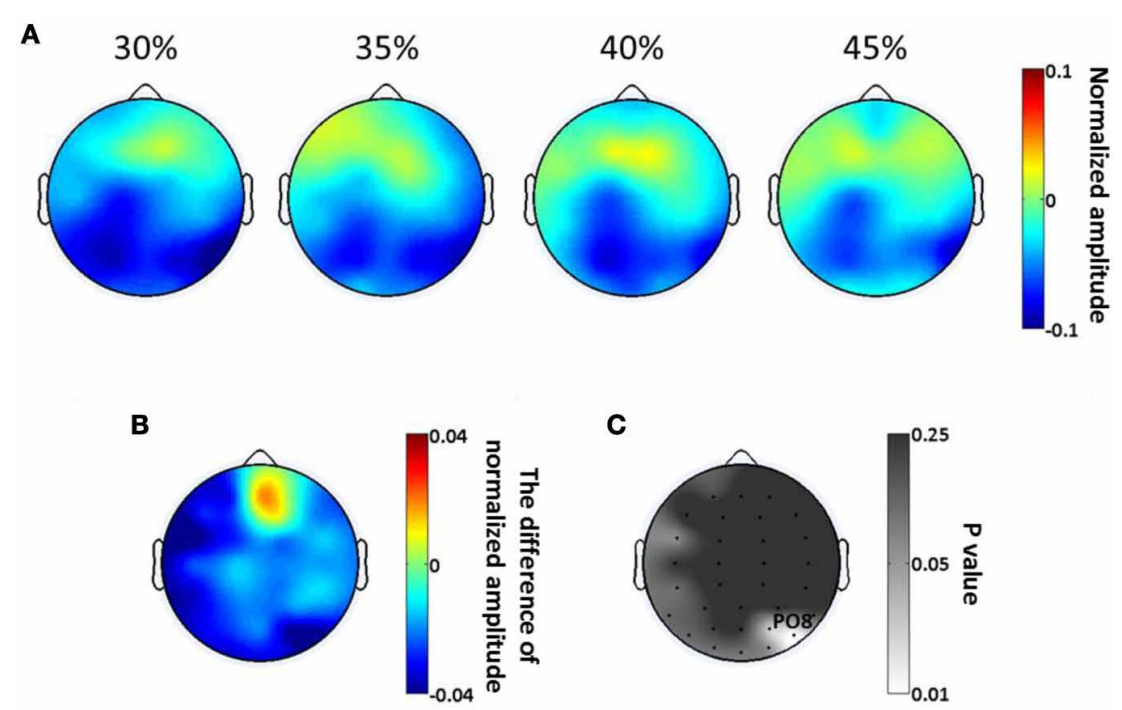

FIGURE 5 | Spatial distribution of exogenous oscillatory modulations,

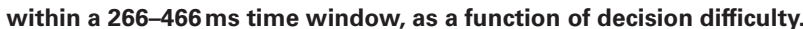
(A) Scalp topographies showing the scalp distribution of average SSVEP amplitude for the four phase coherence (i.e., difficulty) levels. (B) The average difference in SSVEP amplitude between phase coherence levels of 30 and

$45 \%(30-45 \%)$. (C) The p-values at each electrode location, assessing the average SSVEP amplitudes via a paired $t$-test between phase coherence levels of 30 and $45 \%$. Lower p-values indicate more significant differences in mean SSVEP amplitude modulations between the 30 and $45 \%$ phase coherence levels.

electrode location, as assessed by a paired $t$-test, is plotted in Figure 5C. In occipital areas, only electrodes around PO8 show significant effects $(p<0.05)$ of phase coherence/task difficulty.

\section{EFFECT OF PHASE COHERENCE ON ENDOGENOUS OSCILLATIONS}

Changes in normalized endogenous alpha oscillations at electrode PO8 are shown in Figure 6A. The alpha amplitude first increase in the $250 \mathrm{~ms}$ time period after the stimulus, and then falls below the baseline. However, only the time period from 397 to $731 \mathrm{~ms}$ shows a significant difference between phase coherence levels of 30 and $45 \%$, as assessed by a paired $t$-test $(p<0.05)$ and cluster-level statistics $(p<0.05)$. The average suppression in this time period increases with increasing phase coherence levels, which is the 
A

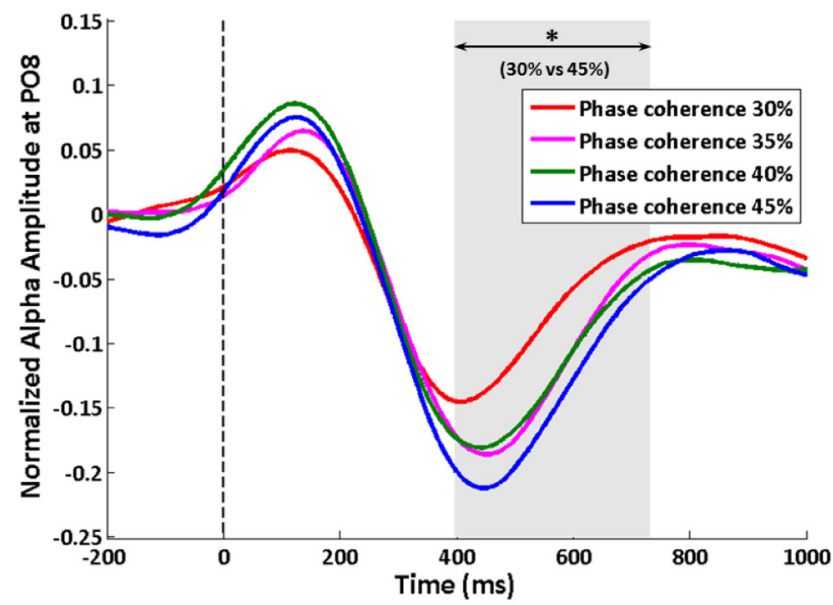

B

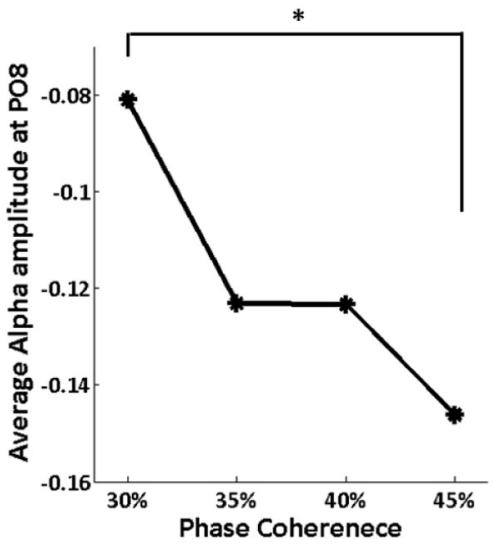

FIGURE 6 | Effect of decision difficulty on endogenous oscillations as measured by normalized alpha amplitude at electrode P08. (A) Time courses of normalized alpha amplitude, shown for each of four phase coherence levels. The shaded area indicates the time period (397-731 ms) having a significant difference in normalized alpha amplitudes between phase coherence levels of 30 and $45 \%$ as assessed by paired $t$-test across subjects and cluster-level statistics ( $p<0.05$ ). The vertical dashed line indicates the onset of task images. (B) The average alpha amplitude from 397 to $731 \mathrm{~ms}$ at each phase coherence level. Asterisks indicate significant differences (paired t-test, $p<0.05)$.
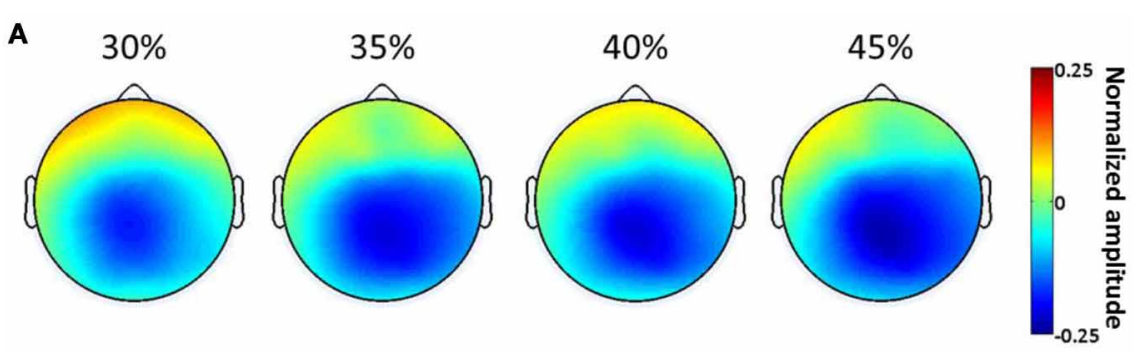

B
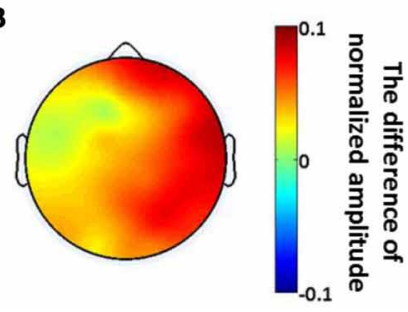

FIGURE 7 | Spatial distribution of endogenous oscillatory modulations, within $\mathbf{3 9 7 - 7 3 1} \mathrm{ms}$ time window, as a function of decision difficulty. (A) Scalp topographies showing the scalp distribution of average alpha amplitude for the four phase coherence (i.e., difficulty) levels. (B) The power difference of average alpha amplitude between phase coherence
C

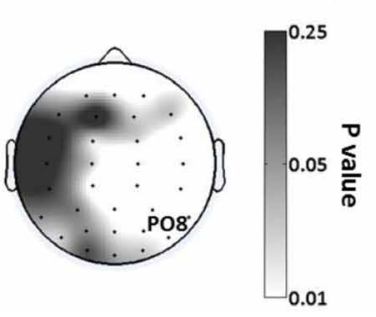

levels of 30 and $45 \%(30-45 \%)$. (C) The $p$-values at each electrode location, assessing the average alpha amplitudes via a paired $t$-test between phase coherence levels of 30 and $45 \%$. Lower p-values indicate more significant differences in mean alpha amplitude modulations between the 30 and $45 \%$ phase coherence levels. reverse of the difficulty modulation on exogenous oscillations (Figure 6B). A significant difference was only found between 30 and $45 \%$ phase coherence levels (paired $t$-test: $t_{(10)}=3.36$, $p=0.007)$.

As in our analysis of SSVEP, we calculated the scalp distributions of average alpha amplitude in the significant time period (397-731 ms) at each phase coherence level (Figure 7A).
Alpha suppression is centered in parietal and occipital regions, with suppression being greater at high phase coherence levels. By plotting the difference in alpha power between 30 and 45\% phase coherence levels and the scalp maps of $p$-values, as shown in Figures 7B and C, we can see that the modulation of the alpha power by phase coherence/task difficulty appears in right lateral parietal-occipital regions. 
A

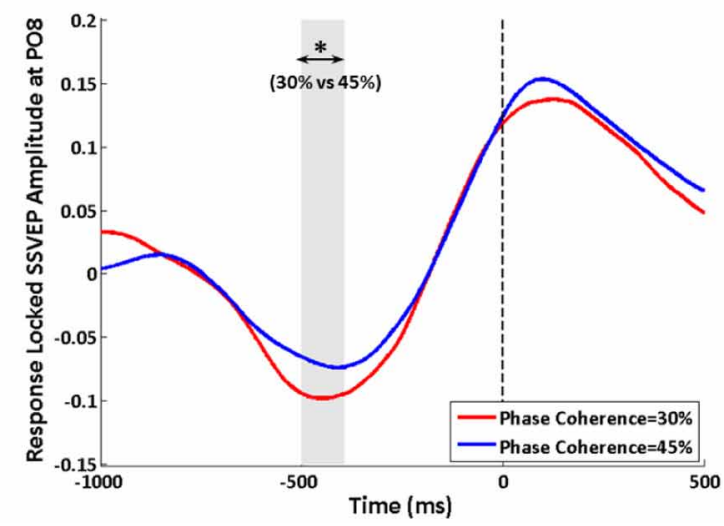

FIGURE 8 | Effects of decision difficulty on response-locked data. (A) SSVEP and (B) alpha oscillations at electrode PO8. The shaded area indicates the time period that shows a significant difference in amplitudes between
B

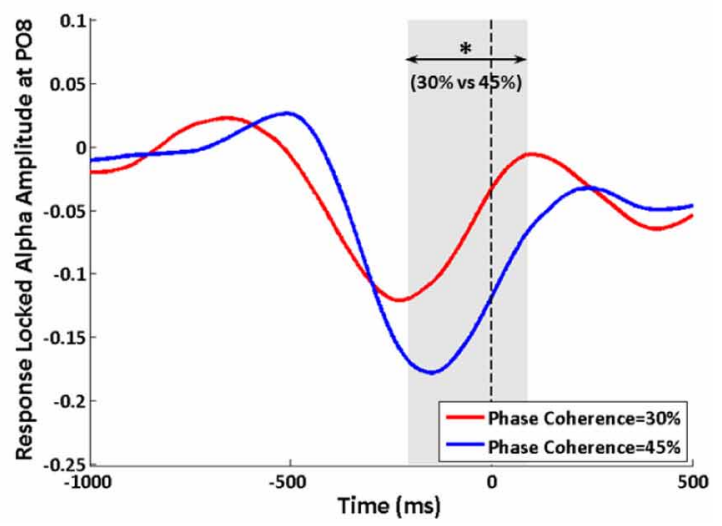

phase coherence levels of 30 and $45 \%$. The vertical dashed lines were locked with reaction time. Asterisks indicate significant differences (paired $t$-test, $p<0.05)$.

\section{EFFECT OF THE MOTOR RESPONSE}

When analyzing the data by aligning trials according to their reactions times, effects of decision difficulty on exogenous induced SSVEP and endogenous alpha oscillations can still be observed between the highest and lowest phase coherence levels. Similar to the stimulus-locked analysis, a lower SSVEP power for 30\% phase coherence trials at electrode PO8 is observed roughly from -500 to $-400 \mathrm{~ms}$ before the response (Figure 8A), while a reversed effect of task difficulty on endogenous alpha oscillations is seen lasting from approximately $-200 \mathrm{~ms}$ before the response until $100 \mathrm{~ms}$ after the response (Figure 8B). The timing of the period that showed a significant difference between difficulty levels was consistent with results of the average reaction time and stimulus-locked analysis. However, the duration was shorter comparing with the corresponding stimulus-locked analysis.

\section{DISCUSSION}

The results of the present study demonstrate that task difficulty, represented here via manipulation of the phase coherence of the visual stimulus, modulates the amplitudes of exogenously induced SSVEPs and endogenous alpha oscillations in different ways and at different times. In our experiment, the amplitude modulations of SSVEP are in response to the task-irrelevant $15 \mathrm{~Hz}$ flickers, while endogenous alpha oscillations reflect the response of an intrinsic rhythm to the face vs. car decision. To compare the effects of task difficulty on SSVEP and endogenous alpha oscillations, we will now summarize several of our observations. First, SSVEP amplitude after the stimulus is immediately decreased, while there is a delay of $250 \mathrm{~ms}$ before suppression of alpha amplitude. Second, the time periods reflecting significant modulation by task difficulty are 266-466 ms and 379-731 ms for SSVEP and alpha oscillations respectively. Third, the correlation between the phase coherence level and the amplitude suppression for SSVEP is opposite to what is seen for alpha. With the increase of the phase coherence level, the reduction of SSVEP amplitude is decreased, while the reduction of alpha amplitude is increased. Fourth, the
SSVEP suppression appears in occipital regions, and the difficulty effect is most significant near electrode PO8. Alpha changes, conversely, are widespread over a broader region in parietal and occipital areas though the most significant modulation of alpha suppression by decision difficulty is primarily in right lateral parietal-occipital areas.

Our findings showed that the suppression of SSVEP amplitude from 266 to $466 \mathrm{~ms}$ was greater at lower phase coherence levels. SSVEP can be used as an objective measure of the allocation of attentional resources (Vialatte et al., 2010 review). The facilitation and suppression of the SSVEP response to the attended and ignored stimuli, respectively, was reported in a study by Andersen and Müller (2010). Previous work using exogenous frequency tagging to investigate to what extent emotional pictures bias competition for attentional processing resources reported a larger decrease in SSVEP amplitude when visual processing load was increased (Müller et al., 2008, 2011; Attar et al., 2010). In addition, previous studies have suggested that additional attentional resources are needed to maintain decision accuracy when the task becomes more difficult (Binder et al., 2004; Grinband et al., 2006; Philiastides et al., 2006; Heekeren et al., 2008 review). Therefore, our observation that there is a greater reduction of SSVEP amplitude for difficult decisions can be interpreted as a neural correlate of this competition for attentional processing resources and supports the hypothesis of the need for more attentional resources when making a difficult decision. Furthermore, this greater SSVEP suppression at low coherence levels is consistent with the perceptual load hypothesis, which says that increased perceptual difficulty of a visual task attenuates the processing of task-irrelevant, unattended distractor stimuli (Yi et al., 2004; Lavie, 2005).

Our analysis showed that normalized SSVEP amplitudes mainly decreased in occipital regions, coinciding with areas that had strong SSVEP responses (Regan, 1989; Vialatte et al., 2010 review). We also found SSVEP suppression in electrodes in close proximity to PO8 exhibited the most significant difficulty effect in our face-car discrimination task. This is consistent with previous 
findings that rLOC is involved in face discrimination and related to decision difficulty for this type of task (Bentin et al., 1996; Jeffreys, 1996; Kanwisher et al., 1997; Liu et al., 2000; Philiastides and Sajda, 2007).

In our study, the suppression of alpha oscillations appeared in parietal-occipital areas, and this topographic distribution of alpha desychronization has been previously reported (Klimesch et al., 1997; Doppelmayr et al., 2002). The right hemisphere in parietal-occipital regions was significantly affected by decision difficulty (Figure 7), which is possibly related to the right hemisphere dominance for face perception (Rhodes, 1985; Luh et al., 1991; Yovel et al., 2008). Object recognition studies found that alpha desynchronization over the right lateral occipital regions exhibited maximal values (Freunberger et al., 2008) and correlated with the proportion of correct object detections (Vanni et al., 1996). Thus both the endogenous and exogenous effects yield scalp topologies that are consistent with the task, suggesting that the oscillations themselves are linked to specific cortical areas involved in processing the stimuli and making the decision.

Similarly to Klimesch et al. (2007 review), the time course of endogenous alpha oscillations in our study showed a synchronization at $250 \mathrm{~ms}$ post-stimulus and a desynchronization around $350-750 \mathrm{~ms}$. The alpha suppression showed the most significant difficulty effect in the time period of $379-731 \mathrm{~ms}$, as it occurred during the time of the late component and motor response previously identified for this type of face-car discrimination task using single-trial analysis of EEG (Philiastides et al., 2006). Previous studies on object recognition/detection showed that the magnitude of alpha desynchronization was related to semantic information processing, and that the recognition of meaningful objects elicited a larger alpha suppression than that for meaningless objects (Klimesch et al., 1997; Vanni et al., 1997; Mima et al., 2001; Freunberger et al., 2008). In our study, the stimuli at high phase coherence level contained more meaningful information than that at the low phase coherence level, since task difficulty was modulated by the percentage of phase coherence. Accordingly, the pattern of alpha suppression, i.e., larger alpha suppression at high phase coherence levels, may be related to semantic information processing. It is also worth mentioning that a study by Siegel et al. $(2007,2011)$ found that visual gamma oscillations increased with a decrease in task difficulty and that gamma-band enhancement was accompanied by a decrease in alpha-band activity. This finding supports our results showing that decreasing task difficulty further suppresses alpha oscillations.

In this study, the difficulty of the discrimination task was manipulated by varying the phase coherence in the images. A study by Bankó et al. (2011) proposed that the effect of phase noise on sensory processing should be dissociated from overall decision difficulty, and suggested that the difficulty component seen in Philiastides et al. (2006) was merely the noise-induced modulation of a bottom-up P2 visual component. However, (Philiastides et al., 2006) explicitly used a modified face vs. car discrimination experiment using colorized images to show that the effect was on decision difficulty since it was present even while keeping the stimulus unchanged, thus reflecting that this difficulty component clearly was also indexing top-down task related processes and was not simply a function of the sensory noise. Our results are interpretable within the context of "taskdifficulty" regardless of whether it is defined bottom-up by the stimulus signal-to-noise and/or top-down by the task.

Additionally, the results from a response-locked analysis showed similar waveforms for the highest and lowest phase coherence levels regardless of the systematic latency differences due to different reaction times for the different difficulty levels (Figure 8). Though the endogenous alpha oscillations in parietaloccipital areas might include a superposition of mu rhythms due to volume conduction and the significant effect for endogenous alpha activity was widespread over a region in right lateral parietal-occipital areas (Figures 7B,C), near to the motor cortex, our results from the response-locked analysis showed a significant modulation of the endogenous oscillations by task difficulty.

A potentially alternative interpretation of our work is that the phase coherence manipulation we employ creates a diffuse partial occlusion that drives processes associated with perceptual closure. Though fragmented line drawings and not phase coherence are usually used to investigate perceptual closure (Doniger et al., 2000; Sehatpour et al., 2008), it is possible that the effects we observe are the same one might see in a perceptual closure experiment. Also intriguing is the similarity in the timing of the difficulty component at $220 \mathrm{~ms}$ (Philiastides et al., 2006) and the Ncl closure component at $250 \mathrm{~ms}$ (Doniger et al., 2000). Though the phase coherence manipulation effects behavioral accuracy and reaction time in a way that one would expect if directly affecting difficulty, further work is needed to investigate whether the manipulation is specific to the subject needing to perceptually close a stimulus for recognition or if it acts on more general processes of evidence accumulation in a noisy stimulus.

In summary, using our experimental paradigm we were able to analyze SSVEP and endogenous alpha oscillations simultaneously while subjects performed a face-car discrimination task where the difficulty of the decision was varied on a trial-to-trial basis. We found that the SSVEP reduction was positively correlated with decision difficulty, while the correlation between the suppression of endogenous alpha oscillations and decision difficulty was negative. The findings indicate that the amplitude modulation of SSVEP reflects the competition for the attentional processing resources and support the hypothesis of the need for more attentional resources when making a difficult decision, while endogenous alpha oscillations may be related to semantic information processing. Lastly, our results demonstrate that exogenous and endogenous oscillations can be simultaneously measured to track the changes in ongoing stimulus-driven and endogenous activities during visual discriminations of varying difficulty.

\section{ACKNOWLEDGMENTS}

This work was support by Grants from NIH (R01- MH085092), the Army Research Office (W911NF-11-1-0219), the National Basic Research Program (973) of China (No. 2011CB933204), the National Natural Science Foundation of China (No. 90820304, No. 91120007), and the Chinese 863 Project (No. 2012AA011601). 


\section{REFERENCES}

Adrian, E. D., and Matthews, B. H. C. (1934). The Berger rhythm: potential changes from the occipital lobes in man. Brain 57, 355-385.

Andersen, S. K., and Müller, M. M. (2010). Behavioral performance follows the time course of neural facilitation and suppression during cued shifts of feature-selective attention. Proc. Natl. Acad. Sci. U.S.A. 107, 13878-13882.

Attar, C. H., Andersen, S. K., and Müller, M. M. (2010). Time course of affective bias in visual attention: convergent evidence from steadystate visual evoked potentials and behavioral data. Neuroimage 53, 1326-1333.

Babiloni, C., Vecchio, F., Bultrini, A., Luca Romani, G., and Rossini, P. M. (2006). Pre- and poststimulus alpha rhythms are related to conscious visual perception: a highresolution EEG study. Cereb. Cortex 16, 1690-1700.

Bankó, E. M., Gal, V., Kortvelyes, J., Kovacs, G., and Vidnyanszky, Z. (2011). Dissociating the effect of noise on sensory processing and overall decision difficulty. J. Neurosci. 31, 2663-2674.

Bentin, S., Allison, T., Puce, A., Perez, E., and McCarthy, G. (1996). Electrophysiological studies of face perception in humans. J. Cogn. Neurosci. 8, 551-565.

Binder, J. R., Liebenthal, E., Possing, E. T., Medler, D. A., and Ward, B. D. (2004). Neural correlates of sensory and decision processes in auditory object identification. Nat. Neurosci. 7, 295-301.

Dakin, S. C., Hess, R. F., Ledgeway, T., and Achtman, R. L. (2002). What causes nonmonotonic tuning of fMRI response to noisy images? Curr. Biol. 12, R476-R477.

Doniger, G. M., Foxe, J. J., Murray, M. M., Higgins, B. A., Snodgrass, J. G., Schroeder, C. E., et al. (2000). Activation timecourse of ventral visual stream object-recognition areas: high density electrical mapping of perceptual closure processes. J. Cogn. Neurosci. 12, 615-621.

Doppelmayr, M., Klimesch, W., Stadler, W., Pollhuber, D., and Heine, C. (2002). EEG alpha power and intelligence. Intelligence 30, 289-302.

Foxe, J. J., and Snyder, A. C. (2011). The role of alpha-band brain oscillations as a sensory suppression mechanism during selective attention. Front. Psychology 2:154. doi: 10.3389/fpsyg.2011.00154
Freunberger, R., Klimesch, W., Griesmayr, B., Sauseng, P., and Gruber, W. (2008). Alpha phase coupling reflects object recognition. Neuroimage 42, 928-935.

Gomez-Ramirez, M., Kelly, S. P., Molholm, S., Sehatpour, P., Schwartz, T. H., and Foxe, J. J. (2011). Oscillatory sensory selection mechanisms during intersensory attention to rhythmic auditory and visual inputs: a human electrocorticographic investigation. J. Neurosci. 31, 18556-18567.

Grinband, J., Hirsch, J., and Ferrera, V. P. (2006). A neural representation of categorization uncertainty in the human brain. Neuron 49, 757-763.

Hanslmayr, S., Aslan, A., Staudigl, T., Klimesch, W., Herrmann, C. S., and Bauml, K. H. (2007). Prestimulus oscillations predict visual perception performance between and within subjects. Neuroimage 37, 1465-1473.

Heekeren, H. R., Marrett, S., and Ungerleider, L. G. (2008). The neural systems that mediate human perceptual decision making. Nat. Rev. Neurosci. 9, 467-479.

Jeffreys, D. A. (1996). Evoked potential studies of face and object processing. Vis. Cogn. 3, 1-38.

Kanwisher, N., McDermott, J., and Chun, M. M. (1997). The fusiform face area: a module in human extrastriate cortex specialized for face perception. J. Neurosci. 17, 4302-4311.

Kelly, S. P., Lalor, E. C., Reilly, R. B., and Foxe, J. J. (2006). Increases in alpha oscillatory power reflect an active retinotopic mechanism for distracter suppression during sustained visuospatial attention. J. Neurophysiol. 95, 3844-3851.

Klimesch, W., Doppelmayr, M., Pachinger, T., and Russegger, $\mathrm{H}$. (1997). Event-related desynchronization in the alpha band and the processing of semantic information. Cogn. Brain Res. 6, 83-94.

Klimesch, W., Sauseng, P., and Hanslmayr, S. (2007). EEG alpha oscillations: the inhibition-timing hypothesis. Brain Res. Rev. 53, 63-88.

Lavie, N. (2005). Distracted and confused?: selective attention under load. Trends Cogn. Sci. 9, 75-82.

Liu, J., Higuchi, M., Marantz, A., and Kanwisher, N. (2000). The selectivity of the occipitotemporal M170 for faces. Neuroreport 11, 337-341.

Lopes da Silva, F. (1991). Neural mechanisms underlying brain waves: from neural membranes to networks. Electroencephalogr. Clin. Neurophysiol. 79, 81-93.
Luh, K. E., Rueckert, L. M., and Levy, J. (1991). Perceptual asymmetries for free viewing of several types of chimeric stimuli. Brain Cogn. 16, 83-103.

Maris, E., and Oostenveld, R. (2007). Nonparametric statistical testing of EEG- and MEG-data. J. Neurosci. Meth. 164, 177-190.

Mima, T., Oluwatimilehin, T., Hiraoka T., and Hallett, M. (2001). Transient interhemispheric neuronal synchrony correlates with object recognition. J. Neurosci. 21, 3942-3948.

Müller, M. M., Andersen, S. K., and Hindi Attar, C. (2011). Attentional bias to briefly presented emotional distractors follows a slow time course in visual cortex. J. Neurosci. 31, 15914-15918.

Müller, M. M., Andersen, S. K., and Keil, A. (2008). Time course of competition for visual processing resourcesbetweenemotionalpictures and foreground task. Cereb. Cortex $18,1892-1899$.

Niedermeyer, E., and Lopes da Silva, F. H. (1993). Electroencephalography: Basic Principles, Clinical Applications, and Related Fields, 3rd Edn. Baltimore, MD: Williams and Williams.

Palva, S., and Palva, J. M. (2007). New vistas for alpha-frequency band oscillations. Trends Neurosci. 30 150-158.

Pfurtscheller, G., Stancak, A., and Neuper, C. (1996). Event-related synchronization (ERS) in the alpha band - an electrophysiological correlate of cortical idling: a review. Int. J. Psychophysiol. 24, 39-46.

Philiastides, M. G., Ratcliff, R., and Sajda, P. (2006). Neural representation of task difficulty and decision making during perceptual categorization: a timing diagram. J. Neurosci. 26, 8965-8975.

Philiastides, M. G., and Sajda, P. (2006) Temporal characterization of the neural correlates of perceptual decision making in the human brain. Cereb. Cortex 16, 509-518.

Philiastides, M. G., and Sajda, P. (2007) EEG-informed fMRI reveals spatiotemporal characteristics of perceptual decision making. J. Neurosci. 27, 13082-13091.

Picton, T. W., John, M. S., Dimitrijevic, A., and Purcell, D. (2003). Human auditory steady-state responses. Int J. Audiol. 42, 177-219.

Regan, D. M. (1989). Brain Electrophysiology: Evoked Potentials and Evoked Magnetic Fields in Science and Medicine. New York, NY: Elsevier.

Rhodes, G. (1985). Lateralized processes in face recognition. Br. J. Psychol. 76, 249-271.
Rihs, T. A., Michel, C. M., and Thut, G. (2007). Mechanisms of selective inhibition in visual spatial attention are indexed by alpha-band EEG synchronization. Eur. J. Neurosci. 25, 603-610.

Rohenkohl, G., and Nobre, A. C. (2011). Alpha oscillations related to anticipatory attention follow temporal expectations. J. Neurosci. 31, 14076-14084.

Sehatpour, P., Molholm, S., Schwartz, T. H., Mahoney, J. R., Mehta, A. D., Javitt, D. C., et al. (2008). A human intracranial study of longrange oscillatory coherence across a frontal-occipital-hippocampal brain network during visual object processing. Proc. Natl. Acad. Sci. U.S.A. 105, 4399-4404.

Siegel, M., Donner, T. H., Oostenveld, R., Fries, P., and Engel, A. K. (2007). High-frequency activity in human visual cortex is modulated by visual motion strength. Cereb. Cortex 17, 732-741.

Siegel, M., Engel, A. K., and Donner, T. H. (2011). Cortical network dynamics of perceptual decisionmaking in the human brain. Front. Hum. Neurosci. 5:21. doi: 10.3389/fnhum.2011.00021

Troje, N. F., and Bülthoff, H. H. (1996). Face recognition under varying poses: the role of texture and shape. Vision Res. 36, 1761-1771.

van Dijk, H., Schoffelen, J. M., Oostenveld, R., and Jensen, O. (2008). Prestimulus oscillatory activity in the alpha band predicts visual discrimination ability. J. Neurosci. 28, 1816-1823.

Vanni, S., Revonsuo, A., and Hari, R. (1997). Modulation of the parietooccipital alpha rhythm during object detection. J. Neurosci. 17, 7141-7147.

Vanni, S., Revonsuo, A., Saarinen, J., and Hari, R. (1996). Visual awareness of objects correlates with activity of right occipital cortex. Neuroreport 8, 183-186.

Vialatte, F. B., Maurice, M., Dauwels, J., and Cichocki, A. (2010). Steadystate visually evoked potentials: focus on essential paradigms and future perspectives. Prog. Neurobiol. 90, 418-438.

Worden, M. S., Foxe, J. J., Wang, N., and Simpson, G. V. (2000). Anticipatory biasing of visuospatial attention indexed by retinotopically specific alpha-band electroencephalography increases over occipital cortex. J. Neurosci. 20, RC63.

Yi, D. J., Woodman, G. F., Widders, D., Marois, R., and Chun, M. M. (2004). Neural fate of ignored 
stimuli: dissociable effects of perceptual and working memory load. Nat. Neurosci. 7, 992-996.

Yovel, G., Tambini, A., and Brandman, T. (2008). The asymmetry of the fusiform face area is a stable individual characteristic that underlies the left-visual-field superiority for faces. Neuropsychologia 46, 3061-3068.

Conflict of Interest Statement: The authors declare that the research was conducted in the absence of any commercial or financial relationships that could be construed as a potential conflict of interest.
Received: 09 November 2012; accepted: 09 January 2013; published online: 31 January 2013.

Citation: Li Y, Lou B, Gao $X$ and Sajda P (2013) Post-stimulus endogenous and exogenous oscillations are differentially modulated by task difficulty. Front. Hum. Neurosci. 7:9. doi: 10.3389/ fnhum.2013.00009
Copyright (c) $2013 \mathrm{Li}$, Lou, Gao and Sajda. This is an open-access article distributed under the terms of the Creative Commons Attribution License, which permits use, distribution and reproduction in other forums, provided the original authors and source are credited and subject to any copyright notices concerning any third-party graphics etc. 